\title{
"Saved by the Pacing Catheter": Gigantic Right Atrial Thromboembolus as a Cause of Pulmonary Embolism
}

\author{
Ana Isabel Costa, Joana Jacinto, Tiago Freitas, Pedro Freitas, Maria Luz Brazão
} Internal Medicine Department, Central Hospital of Funchal SESARAM, Funchal, Portugal

Received: 03/01/2015

Accepted: 19/01/2015

Published: $23 / 02 / 2015$

How to cite this article: Costa Al, Jacinto J, Freitas T, Freitas P, Brazão ML. "Saved by the pacing catheter": gigantic right atrial thromboembolus as a cause of pulmonary embolism. EJCRIM 2015;2:doi: 10.12890/2015_000176

Conflicts of Interests: The authors declare that they have no conflicts of interest in this research

This article is licensed under a Commons Attribution Non-Commercial 4.0 License

\section{ABSTRACT}

Pulmonary embolism (PE) related to the presence of right heart thromboemboli entails a higher mortality rate than PE alone. Furthermore, right heart thromboemboli are often associated with deep venous thrombosis. The most effective therapy for haemodynamically stable patients remains unknown, although recent data suggest that thrombolytic therapy is associated with a better outcome. We describe the case of an 83-year-old woman, hospitalized with PE consequent to right heart thrombus-in-transit, in whom investigation revealed a concomitant deep venous thrombosis. She required thrombolysis, given the high mortality risk that is traditionally associated with this clinical entity.

\section{LEARNING POINTS}

- Pulmonary embolism secondary to right-sided cardiac thromboembolus entails a higher mortality rate than PE alone.

- Echocardiography is useful for the rapid detection of cardiac thrombi in patients with suspected PE.

- There is no consensus on the best therapeutic approach in this context, although there is some evidence that supports thrombolysis.

\section{KEYWORDS}

Pulmonary embolism, right heart thromboembolus, deep venous thrombosis, thrombolysis

\section{INTRODUCTION}

Right intracardiac thrombi are rare events. Nevertheless, they do have important diagnostic and therapeutic implications in patients with pulmonary embolism (PE) ${ }^{[1]}$. Predisposing factors include indwelling wires/catheters, dilated cavities, atrial fibrillation (Afib), prothrombotic states and other potential embolic sources. Ante-mortem diagnosis was unusual before the advent of echocardiography, being first described by Covarrubias et al. ${ }^{[2]}$ in 1977. Right-sided cardiac intracavitary thromboembolus, unlike local thrombosis, consists of embolic material that is temporarily lodged in the right atrium or ventricle, a condition frequently associated with deep venous thrombosis. Also known as "embolus in transit", it is often depicted as a highly mobile mass, with a serpentine shape, that wanders inside the right atrium and/ or ventricle, sometimes prolapsing through the tricuspid and/or pulmonary valves during the cardiac cycle. The embolus' fixation point is normally seen as a narrow pedicle. The echocardiographic characteristics are important to distinguish it from a mural thrombus, which has less mobility, a wider implantation base and the occasional presence of small focal calcifications ${ }^{[2,3]}$.

The true incidence is not known, although it is estimated to be between $3 \%$ and $23 \%$ in patients with PE. Right atrium thromboembolusrelated $\mathrm{PE}$ is associated with a higher mortality rate $(30 \%)$ than $\mathrm{PE}$ alone $(8 \%)^{[2,3,4]}$.

Despite this evidence, there is no consensus on the best therapeutic approach. The largest retrospective study comparing the three different options (anticoagulation, surgery and thrombolysis) involved 177 cases of cardiac right thromboemboli, $98 \%$ of which presented with PE. The global mortality rate was $27.1 \%$; however, patients submitted to thrombolysis had a higher survival rate $(p<0.05)$ than those treated with anticoagulation or surgical therapies. The major complication reported was bleeding ${ }^{[5]}$. Furthermore, a prospective trial conducted by Pierre-Justin and Pierard ${ }^{[4]}$ concluded that, although the incidence of right cardiac thrombi is low in patients admitted with PE, thrombolytic therapy with recombinant tissue plasminogen activator (rt-PA) seems to be rapidly efficient in improving pulmonary perfusion in the subgroup of patients with PE secondary to right heart thromboembolus.

Hence, there is data supporting thrombolytic therapy as the first-line treatment in this context.

\section{CASE REPORT}

The patient was an 83-year-old Caucasian woman, with a medical history of hypertension, ischemic cardiomyopathy and Afib. She had had a VVI pacemaker for 10 years. She presented in the emergency room (ER) complaining of a twinge-like left hemithoracic pain, with pleuritic 


\section{Laboratory findings}

Elevation of inflammatory markers: leucocytosis with neutrophilia and increased C-reactive protein (CRP)

High D-dimer $(>5.0 \mu \mathrm{g} / \mathrm{ml})$

Arterial blood gas analysis (room air): hypoxaemia and hypocapnia $\left(\mathrm{SatO}_{2} 90 \%, \mathrm{pO}_{2} 69 \mathrm{mmHg}, \mathrm{pCO}_{2} 30 \mathrm{mmHg}\right.$ )

Table 1: Laboratory findings

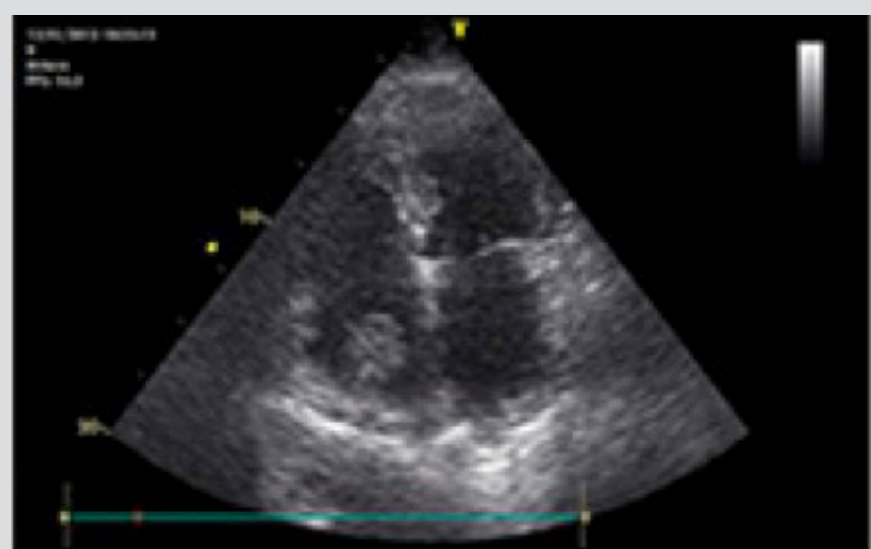

Figure 1: Thromboembolus in the right atrium, adhering to the pacing catheter.

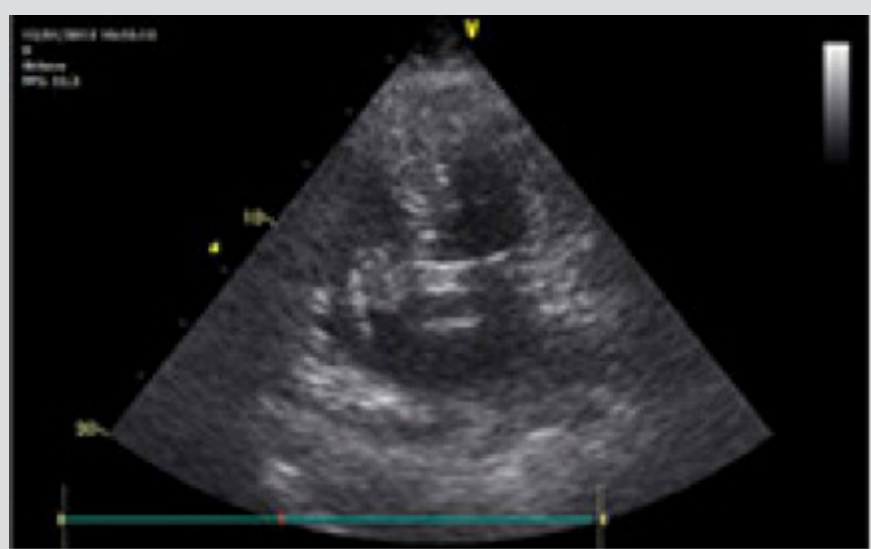

Figure 2: Mobile thromboembolus, serpentinuous, prolapsing through the tricuspid valve.

characteristics, emphasizing having suffered fatigue for months, which had worsened during the previous week. She denied fever, cough or other complaints. On admission: afebrile, slight dyspnoea, blood pressure (BP) 160/90 mmHg, irregular pulse around 90 bpm; cardiac auscultation revealed an aortic grade II/VI intensity mesosystolic murmur. Concerning laboratory findings are listed in Table 1.

Transthoracic echocardiogram showed mild aortic valvular stenosis (fibrodegenerative), bilateral atrial dilatation, good left ventricular systolic function and a normal right ventricle. A bulky image was visible on the right atrium, suggestive of a thrombus; it was mobile and adherent to the pacing catheter (greatest diameter of $23 \mathrm{~mm}$ ). Pulmonary Artery Systolic Pressure (PASP) was estimated to be $57 \mathrm{mmHg}$ (Figs. 1 and 2).

Results of the contrasted thoracic CT scan were as follows: "Pulmonary artery repletion defects, concerning the left pulmonary artery, thoroughly involving the superior lobe branch and its segmental branches. On the right, there is a small repletion defect of the superior lobe branch and a distal thrombus in the right pulmonary artery that does not cause repletion defect of the inferior or medium lobes."

The patient was admitted to an Intermediate Care Unit and heparinized. A multidisciplinary consultation (including a cardiothoracic surgeon) concluded surgery would have no benefit in this context, given her advanced age and the high surgical risk. By Day 2, the echocardiogram showed no improvement and facing the imminent risk of massive embolization, it was decided to initiate thrombolytic therapy with Alteplase (rtPA). Eight hours later, the patient presented with hypotension, tachycardia and decreased breath sounds on the left side. A $2 \mathrm{~g} /$ dl haemoglobin level drop stood out. Thoracic CT revealed: "Atelectasis of the left lung associated with major left pleural effusion". A chest tube was placed with immediate drainage of $1000 \mathrm{cc}$ of haematic content; volaemic resuscitation with fluids and blood transfusion were successful in restoring haemodynamic stability. Anticoagulation was reinstated with unfractionated heparin, with protocolized activated Partial Thromboplastin Time (aPPT) evaluation.

By Day 3 post-thrombolysis, the echocardiography showed complete resolution of the intracardiac thromboembolus with PASP of 25 $\mathrm{mmHg}$; the patient was stable and the chest tube was removed. On Day 4, a venous echodoppler of the lower limbs revealed deep vein thrombosis on the left femoral and popliteal veins. On Day 5, bridging with warfarin was initiated. During the rest of the hospital stay, the patient progressively began to sit up, remaining stable and asymptomatic. She was discharged on Day 18 and referred to a cardiology appointment, with complete resolution of her illness.

\section{CONCLUSION}

There is no consensus on the therapeutic approach for patients with PE secondary to intracardiac thromboembolus, and the current literature on how to tackle the ideal therapy in this context is scarce: it considers anticoagulation, thrombolysis or surgery. In the case of a haemodynamically unstable patient, thrombolytic therapy is considered the best choice, however there is no agreement when treating an otherwise stable patient. In the reported case, the patient had predisposing factors for cardiac thrombi such as AFib, RA dilation and an indwelling wire. However, the presence of deep venous thrombosis seemed to be the major player, and, in our opinion, if it weren't for the presence of the pacing catheter clutching to those emboli, misfortune would surely have ensued. The thrombus' dimension and the potential risk for massive PE were fundamental determinants in the decision to undertake thrombolytic therapy. 


\section{REFERENCES}

Chartier L, Bera J, Delomez M et al. Free floating thrombi in right heart: diagnosis, management and prognostic indexes in 38 consecutive patients. Circulation 1999;99:2779-2783. Covarrubias EA, Sheikh MU, Fox LM. Echocardiography and pulmonary embolism. Ann Intern Med 1977;87:720.

European Working Group on Echocardiography. The European Cooperative Study on the clinical significance of right heart thrombi. Eur Heart J 1989;10:1046-1059.

Pierre-Justin G, Pierard LA. Management of mobile right heart thrombi: a prospective series. Int J Cardiol 2005;99:381-388.

5. Rose P, Punjabi N, Pearse D. Treatment of right heart thromboemboli. Chest 2002;121:806-814. 